Cite this article as: BMJ, doi:10.1136/bmj.38965.375718.80 (published 13 October 2006)

Research

\title{
Self management of arthritis in primary care: randomised controlled trial
}

Marta Buszewicz, Greta Rait, Mark Griffin, Irwin Nazareth, Anita Patel, Angela Atkinson, Julie Barlow, Andy Haines

\begin{abstract}
Objective To evaluate clinical effectiveness of a self management programme for arthritis in patients in primary care with osteoarthritis.

Design Randomised controlled trial.

Setting 74 general practices in the United Kingdom.

Participants 812 patients aged 50 and over with osteoarthritis of hips or knees (or both) and pain or disability (or both).

Intervention Participants were randomised to six sessions of self management of arthritis and an education booklet (intervention group) or the education booklet alone (control group).
\end{abstract}

Main outcome measures Primary outcome was quality of life, as assessed by the short form health survey (SF-36). Several other physical and psychosocial secondary outcomes were assessed. Data were collected at baseline, four months, and 12 months.

Results Response rates were $80 \%$ and $76 \%$ at four and 12 months. The two groups showed significant differences at 12 months on the anxiety subscore of the hospital anxiety and depression scale (mean difference $-0.62,95 \%$ confidence interval -1.08 to -0.16 ), arthritis self efficacy scale for pain (0.98, 0.07 to 1.89$)$, and self efficacy for other aspects of management (1.58, 0.25 to 2.90$)$. Results were similar for intention to treat and per protocol analyses. No significant difference was seen in number of visits to the general practitioner at 12 months.

Conclusions The self management of arthritis programme reduced anxiety and improved participants' perceived self efficacy to manage symptoms, but it had no significant effect on pain, physical functioning, or contact with primary care.

Trial registration Current Controlled Trials ISRCTN79115352.

\section{Introduction}

Osteoarthritis is a common chronic disease associated with ageing and a major worldwide problem for medical, psychosocial, and economic reasons. ${ }^{1}$ At least five million UK adults (8\% of the population) have moderate to severe osteoarthritis. ${ }^{2}$ Affected people are mainly cared for in the community.

Osteoarthritis leads to considerable morbidity in terms of pain, functional disability, lowered quality of life, and psychological problems. ${ }^{1}$ Patient centred programmes for self management of arthritis, tested in the United States on volunteers from the community with osteoarthritis and rheumatoid arthritis, had beneficial effects on pain, depression, exercise taken, communication with doctors, and participants' perception of their capac- ity to manage the disease (arthritis self efficacy). ${ }^{3}$ Similar results were found in studies on volunteers in the UK. ${ }^{4}$ A review commissioned by the Department of Health suggested that self management can improve knowledge, performance of self management behaviours, self efficacy, and aspects of health status compared with standard care, but the studies reviewed had small sample sizes and short follow-up, and they used non-validated outcome measures. ${ }^{5}$

A recent US randomised controlled trial of participants from primary care with osteoarthritis, rheumatoid arthritis, or fibromyalgia recruited to programmes for self management of arthritis showed no difference in any outcomes measured (disability, pain, self efficacy, mental health, and satisfaction) at four months. ${ }^{6}$ This was a small study of 178 subjects, however, and highlights the need for a large well designed randomised trial in primary care. This is relevant to the UK government's promotion of the expert patient programme in primary care settings, a generic self management programme for people with a variety of chronic diseases (www.expertpatients.nhs.uk/), developed from the arthritis self management programme. We hypothesised that for primary care patients with osteoarthritis, participation in an arthritis self management programme and receipt of an educational booklet would improve their overall function compared with receipt of the booklet alone.

\section{Methods}

\section{Practices}

People with osteoarthritis were recruited from UK general practices in areas where the voluntary organisation Arthritis Care provided the "challenging arthritis" intervention. This is a programme for self management of arthritis developed from the original US model based on social cognitive theory, in which self efficacy plays an important part (see box A on bmj.com). ${ }^{7}$ We identified general practices that belonged to the Medical Research Council's research framework and, in areas where this was not possible, we approached local primary care research networks. Trained research nurses recruited practice participants.

\section{Recruitment}

We recruited participants between December 2000 and February 2003. Twelve month follow-up for all participants ended in March 2004.

Figures $A$ and $B$, figure legends, and boxes 1 and 2 are on bmj.com 


\section{Participants}

Patients were eligible if they were aged 50 or more, had been clinically diagnosed with osteoarthritis of the hips or knees (or both) for at least a year, and had had associated pain or functional disability (or both) during the past month. We excluded people who had been recommended surgery for arthritis or who had poor mobility, poor understanding of English, associated neurological signs, or cognitive impairment.

General practitioners identified potential participants from practice attendees over a six week period. Practices' computerised records were also searched for relevant osteoarthritis clinical terms (Read codes) and prescriptions for regular non-steroidal anti-inflammatory drugs or analgesics. We examined all clinical records to establish the duration of the diagnosis of osteoarthritis. Research nurses posted study details, a screen for current symptoms of osteoarthritis, and an invitation to discuss the trial to potential participants. Patients who met all eligibility criteria and consented to participate were randomised to one of two groups.

\section{Randomisation}

We used an independent centralised computerised system to randomise the participants. Practices were stratified by area and we used minimisation to balance for differences in age and sex. Research nurses faxed details of consenting participants to the trial manager, who passed the information on to the randomisation centre that allocated participants to their experimental group. The trial manager was the only member of the research team not blinded to the allocation.

The intervention group received an education booklet and an invitation to participate in a local challenging arthritis course. The booklet was designed for the study and incorporated information available to the public from Arthritis Care and the Arthritis Research Campaign.

The control group received only the education booklet. This reflects standard primary care, where giving written information is common practice. Research suggests leaflets have substantially less impact than the self management programme on the outcomes measured. ${ }^{8}$

Outcomes were assessed through questionnaires completed by the participants at entry to the trial before randomisation and at four and 12 months (box B on bmj.com). All measures were validated in primary care populations. ${ }^{9-12}$ The four and 12 month questionnaires were sent to all participants with a postage paid return envelope. Non-responders were sent a reminder questionnaire by recorded delivery. At 12 months, nonresponders were telephoned and given the option of completing only the primary outcome measure (the short form health survey, SF-36).

\section{Statistical methods and analysis}

Data were double entered and analysed using SPSS for Windows Release 11.5.0 and SOLAS for missing data analysis version 3.2. We calculated sample size on the basis of the primary outcome measure (SF-36). If the dropout rate was 30\%, 1000 participants would be needed to detect a six point difference in SF-36 with $90 \%$ power and a $5 \%$ two sided significance level, assuming a standard deviation of $25 .{ }^{13}$ The arthritis self efficacy scale was an important secondary measure, and we estimated that 1000 participants would allow us to detect a difference of 2.5 points, assuming a standard deviation of $10 .^{14}$

We used descriptive statistics to outline the characteristics of the trial participants. To adjust for baseline scores, we used analysis of covariance to compare outcome between the two groups. ${ }^{15}$ Assumptions required for this method were found to be valid for

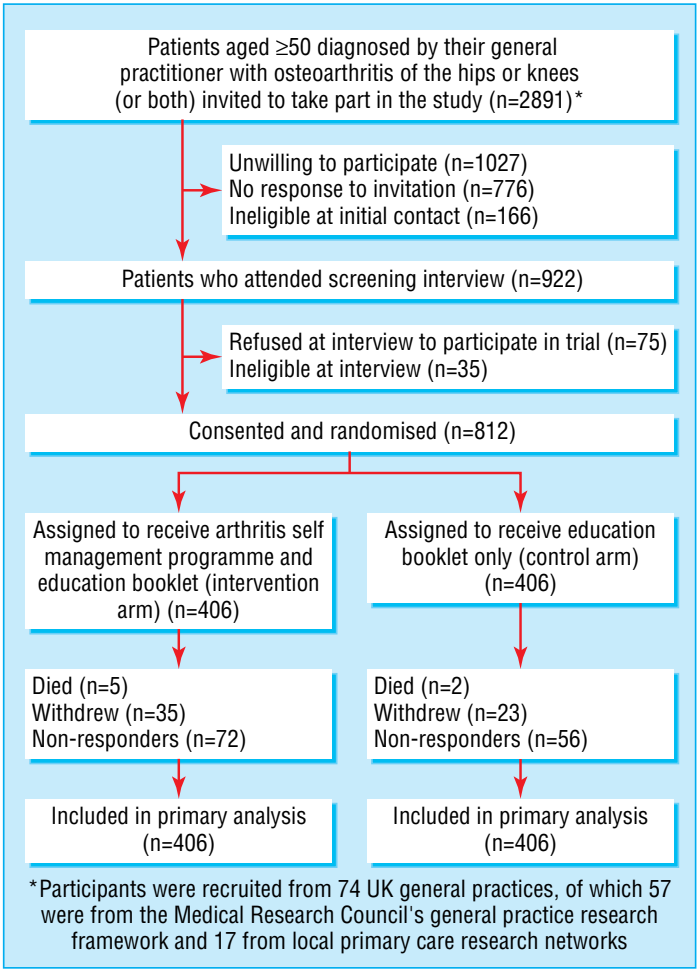

Flow of participants through trial

all outcomes. Primary comparisons assessed the effect of the intervention at 12 months. Analysis was based on intention to treat. We used imputation techniques to assess the impact of missing data. Baseline scores were imputed for participants with missing baseline values, using the technique of "hot decking," based on age, sex, and randomisation group. Once a baseline score was available, we imputed multiple complete data sets (five) using a predictive model based approach with ordinary least squares regression. ${ }^{17}$ We used the standard complete data method (analysis of variance) to analyse each imputed data set. The results were combined using explicit standard formulae to provide results based on imputation of missing data.

For the multiple imputation analysis we split participants into those who attended four or more sessions (judged to have received a clinically significant "dose" ${ }^{\text {") }}$ ) and those who attended fewer than four. (The number of sessions attended was unknown for seven participants, and they were included in the group that attended fewer than four sessions.) We also undertook a per protocol analysis of patients who had attended four or more sessions and for whom full sets of data were available.

All comparisons of outcome scores between intervention and control groups are presented as the difference in means after adjusting for baseline differences in the outcome. We used standard parametric techniques to calculate confidence intervals for these differences.

\section{Economic evaluation}

Service use and cost implications were fully examined in an economic evaluation carried out as part of our study. The results of this will be reported separately.

\section{Results}

We approached 2891 people from 74 UK general practices and recruited 812 participants (figure). Questionnaire response rates 
Table 1 Baseline characteristics of patients in a randomised controlled trial of self management of arthritis. Values are number (percentage) unless stated otherwise

\begin{tabular}{|c|c|c|}
\hline \multirow{2}{*}{ Characteristic } & \multicolumn{2}{|c|}{ Group } \\
\hline & Intervention & Control \\
\hline \multicolumn{3}{|l|}{ Age } \\
\hline Mean (SD) & $68.4(8.2)$ & $68.7(8.6)$ \\
\hline \multicolumn{3}{|l|}{ Sex } \\
\hline Male & $151 / 406 \quad(37)$ & $151 / 406 \quad(37)$ \\
\hline Female & $255 / 406(63)$ & $255 / 406 \quad(63)$ \\
\hline \multicolumn{3}{|l|}{ Accommodation } \\
\hline Owner occupier & $323 / 391 \quad(83)$ & $302 / 384$ (79) \\
\hline Staying with family or friends & $3 / 391 \quad(<1)$ & $5 / 384(1)$ \\
\hline Rented accommodation & $65 / 391$ (17) & $77 / 384(20)$ \\
\hline \multicolumn{3}{|l|}{ Ethnic origin } \\
\hline White & $388 / 390(100)$ & $382 / 385$ (99) \\
\hline Black African & $0 / 390(0)$ & $1 / 385(<1)$ \\
\hline Black Caribbean & $2 / 390(<1)$ & $2 / 385(1)$ \\
\hline \multicolumn{3}{|l|}{ Age when left school } \\
\hline Below 16 years & $249 / 390(64)$ & $259 / 383(68)$ \\
\hline 16 to 18 years & $141 / 390(36)$ & $124 / 383(32)$ \\
\hline \multicolumn{3}{|l|}{ Higher education } \\
\hline No & $281 / 388$ (72) & $280 / 382(73)$ \\
\hline Yes & $107 / 388$ (28) & $102 / 382(27)$ \\
\hline
\end{tabular}

were $95 \%$ at baseline, $80 \%$ at four months, and $76 \%$ at 12 months, with no significant differences in response rates between

Table 2 Mean (SD) baseline results of all outcome measures after "hot deck" imputation of missing data in randomised controlled trial of self management of arthritis

\begin{tabular}{|c|c|c|}
\hline \multirow{2}{*}{ Outcome measure } & \multicolumn{2}{|c|}{ Group } \\
\hline & Intervention $(\mathrm{n}=406)$ & Control $(n=406)$ \\
\hline \multicolumn{3}{|l|}{ Short form 36} \\
\hline Summary physical health & $25.4(10.6)$ & $25.1(11.7)$ \\
\hline Summary mental health & $52.2(10.8)$ & $50.6(10.6)$ \\
\hline \multicolumn{3}{|c|}{ WOMAC osteoarthritis index } \\
\hline Pain & $8.8(3.6)$ & $8.7(3.7)$ \\
\hline Stiffness & $3.8(1.6)$ & $3.8(1.6)$ \\
\hline Physical functioning & $28.6(12.3)$ & $29.1(12.7)$ \\
\hline \multicolumn{3}{|c|}{ Hospital anxiety and depression scale } \\
\hline Anxiety subscale & $7.4(4.5)$ & $7.2(4.1)$ \\
\hline Depression subscale & $5.5(3.3)$ & $5.5(3.2)$ \\
\hline \multicolumn{3}{|l|}{ Arthritis self efficacy scale } \\
\hline Pain & $18.9(6.7)$ & $19.2(6.4)$ \\
\hline Other & $25.6(8.2)$ & $25.7(7.7)$ \\
\hline
\end{tabular}

groups. We found no significant differences in patient characteristics at baseline between the intervention and control groups (table 1). The mean age of participants was 68 years, and two thirds $(66 \% ; 510 / 812)$ were women. Most participants were white and owned their homes rather than renting. In the intervention group more than half $(56 \% ; 219 / 392)$ attended four or more sessions, 9\% (37/392) attended only one or two sessions, and 29\% (115/392) attended none.

At baseline we found no significant differences between the groups in outcome variables (table 2). In the intention to treat analysis with imputation of missing values we found no significant differences in the primary outcome (SF-36 physical and mental health scores), but in the intervention group we found a trend towards improvement on the mental health scale (table 3; figs A and B on bmj.com). With respect to the secondary outcomes, we found no significant differences for the three components of the WOMAC osteoarthritis index, although the intervention group showed a consistently favourable trend, which was greater at 12 months than four months.

In the intervention group the anxiety subscore of the hospital anxiety and depression scale (HADS) was significantly reduced at 12 months (adjusted difference in means $-0.62,95 \%$ confidence interval -1.08 to -0.16 ). We also found a significant reduction in the depression subscore at four months $(-0.40$ ( -0.76 to -0.03$)$, but this was lost by 12 months. We found a significant difference between groups for the pain subscore of the arthritis self efficacy (ASE) scale after four and 12 months (1.63, 0.83 to $2.43 ; 0.98,0.07$ to 1.89$)$ and for the "other" subscore (1.83, 0.74 to $2.92 ; 1.58,0.25$ to 2.90 ).

The two groups did not differ significantly in rates of consultation with a general practitioner at 12 months before the study or at 12 months' follow-up. The results of the intention to treat analysis and the per protocol analysis (those patients who completed four or more sessions of the intervention and for whom we had complete data) were similar (table 3).

\section{Discussion}

Our trial adds substantially to the literature on self management of osteoarthritis because of its relatively large size ${ }^{18}$ and because participants were recruited from primary care rather than from outpatient clinics or via advertisements. The intervention had an impact on the psychological wellbeing of participants. It reduced anxiety, as measured by the hospital anxiety and depression scale, and it increased self efficacy-participants were more con-

Table 3 Outcomes of a randomised controlled trial of self management of arthritis

\begin{tabular}{|c|c|c|c|c|}
\hline \multirow{3}{*}{ Outcome measure } & \multicolumn{4}{|c|}{ Adjusted difference in means $(95 \% \mathrm{Cl})$} \\
\hline & \multicolumn{2}{|c|}{4 months } & \multicolumn{2}{|c|}{12 months } \\
\hline & Intention to treat analysis & Per protocol analysis & Intention to treat analysis & Per protocol analysis \\
\hline \multicolumn{5}{|l|}{ Short form 36} \\
\hline Mental health & $0.11(-1.18$ to 1.40$)$ & 0.82 (-0.94 to 2.57) & $1.35(-0.03$ to 2.74$)$ & 1.56 (-0.28 to 3.39$)$ \\
\hline Physical health & $0.22(-1.50$ to 1.94$)$ & $-0.37(-2.02$ to 1.28$)$ & $0.33(-1.31$ to 1.98$)$ & 0.24 (-1.63 to 2.11$)$ \\
\hline \multicolumn{5}{|c|}{ WOMAC osteoarthritis index } \\
\hline Pain & $-0.15(-0.57$ to 0.28$)$ & $-0.30(-0.79$ to 0.19$)$ & $-0.33(-0.78$ to 0.13$)$ & $-0.47(-1.05$ to 0.10$)$ \\
\hline Stiffness & $-0.05(-0.28$ to 0.17$)$ & $-0.12(-0.36$ to 0.11$)$ & $-0.17(-0.43$ to 0.09$)$ & $-0.13(-0.40$ to 0.14$)$ \\
\hline Physical function & $-1.22(-2.59$ to 0.16$)$ & $-0.80(-2.24$ to 0.63$)$ & $-1.17(-2.84$ to 0.50$)$ & $-0.95(-2.63$ to 0.74$)$ \\
\hline \multicolumn{5}{|c|}{ Hospital anxiety and depression scale } \\
\hline Anxiety & $-0.36(-0.76$ to 0.05$)$ & $-0.68(-1.15 \text { to }-0.20)^{*}$ & $-0.62(-1.08 \text { to }-0.16)^{*}$ & $-0.72(-1.24 \text { to }-0.21)^{*}$ \\
\hline Depression & $-0.40(-0.76 \text { to }-0.03)^{\star}$ & $-0.57(-0.96 \text { to }-0.18)^{\star}$ & $-0.41(-0.82$ to 0.01$)$ & $-0.33(-0.76$ to 0.10$)$ \\
\hline \multicolumn{5}{|c|}{ Arthritis self efficacy scale } \\
\hline Pain & $1.63(0.83 \text { to } 2.43)^{*}$ & $2.55(1.56 \text { to } 3.56)^{*}$ & $0.98(0.07 \text { to } 1.89)^{*}$ & $1.43(0.37 \text { to } 2.48)^{*}$ \\
\hline Other & $1.83(0.74 \text { to } 2.92)^{*}$ & $2.81(1.74 \text { to } 3.87)^{*}$ & $1.58(0.25 \text { to } 2.90)^{\star}$ & $1.54(0.48 \text { to } 2.60)^{*}$ \\
\hline
\end{tabular}

${ }^{*}$ Statistically significant. 
fident about managing pain and other arthritis related symptoms as a result.

Other outcomes, in particular the mental health subscale of the SF-36, showed a favourable trend in the intervention group, but this was not statistically significant. These findings indicate that the intervention improves participants' ability to manage their symptoms and leads to a sustained reduction in anxiety, but that it does not substantially affect physical functioning or levels of pain experienced. The intervention did not reduce the number of consultations with a general practitioner; this may be because the intervention encouraged participants to seek advice from their general practitioner. Reductions in consultation rates might arise after the 12 month follow-up, as a result of greater self efficacy.

\section{Comparison with other studies}

We used established and validated clinical measures that have been used in other studies of osteoarthritis. Many studies of self management programmes for arthritis have used other outcome measures, including health behaviours (exercise, management of cognitive symptoms, communication with doctors, use of community resources, and management of mental stress) and health status (fatigue and positive affects). These are usually self reported measures, which are not always validated, and they have indicated an increase in desirable patient behaviours, such as taking more exercise, after the intervention. ${ }^{18}$ It is not clear whether these changes have positive and long term effects on patient behaviour and morbidity. Moreover, only a few studies report improved quality of life. ${ }^{19}$ This range of reported outcomes raises questions about which outcomes are most meaningful socially and clinically, and what are appropriate effect sizes.

A clear indication of the clinical efficacy of self management programmes for arthritis is hard to establish from the literature because of the variability of previous studies, which were heterogeneous in terms of the intervention (group or one to one programmes, of different lengths), underlying theory (cognitive, social construct theory, or neither), and methods. ${ }^{180}$ A recent, comprehensive systematic review of education programmes for self management of chronic disease found small to moderate effects for selected outcomes in chronic diseases (such as diabetes and asthma). Education programmes for self management of arthritis were associated with a trend towards reduced pain and disability, but the effects were not statistically significant. ${ }^{20}$ Psychological outcomes were not examined. Some evidence exists, however, that treating depression in patients with osteoarthritis may help reduce the amount of pain experienced and improve functional status and quality of life. ${ }^{21}$

Most previous studies involved volunteers, and few randomised controlled trials have been carried out. A recent randomised controlled trial of self management programmes for arthritis that studied US patients with osteoarthritis, rheumatoid arthritis, or fibromyalgia recruited from primary care showed no difference in any of the outcomes measured (disability, pain, self efficacy, mental health, or satisfaction) at four months. ${ }^{6}$ This was a small study, however, with a total of 178 participants. Only $12 \%$ of those approached were recruited, and the completed questionnaire response rate was only $57 \%$.

\section{Strengths and limitations}

This was a large randomised controlled trial of the effect of a self management programme for osteoarthritis in a primary care population. Although we did not recruit the initial target of 1000 participants, our attrition rate ( $24 \%$ by 12 months) was lower than the anticipated $30 \%$. Initial sample size calculations were based on SF-36 and arthritis self efficacy scores. Our data showed that initial assumptions about the variability of these measures (standard deviations) were conservative, and thus we had overestimated the sample size needed. The trial would almost certainly have identified clinically meaningful differences in primary outcomes if they existed.

As the study was a pragmatic primary care based trial we recruited participants who had been diagnosed with osteoarthritis by their general practitioner rather than applying formal assessment criteria, which are not used in routine general practice. The sociodemographic data for our participants in terms of housing status was similar to 2001 UK census data, apart from a slight predominance of white participants (99.5\%) compared with the national average of $96 \%$ for this age group. ${ }^{22}$

Almost 30\% of the intervention group did not attend any "challenging arthritis" sessions. Telephone interviews with a subgroup of non-attenders indicated the main reason was timing of the local group or difficulties with access. Results of the intention to treat and per protocol analyses suggest that poor attendance did not affect the final results-results were similar for an intention to treat analysis of all participants who had been offered the intervention and those who attended four or more sessions. Such programmes need to consider how to maximise participation, and the relatively poor uptake of the intervention is of concern in terms of the accessibility and acceptability of self management programmes in primary care.

Our trial results show small positive changes across all outcome measures, but these were statistically significant only for mental health outcomes and arthritis self management behaviours. Patients who volunteered for previous studies may have been more severely affected than those recruited from primary care for this trial. It is difficult to compare baseline values with other self management studies because of variability in the outcome measures used. However, comparison of anxiety and depression using the hospital anxiety and depression scale shows higher mean baseline values in a group of UK volunteers studied than in the primary care patients in our trial. ${ }^{14}$ Despite this, our intervention group still showed a significant improvement in anxiety.

\section{Policy implications}

The UK government has been promoting the large scale provision of the expert patient programme through referral from primary care before a formal evaluation has been completed, ${ }^{23}$ and our results should help inform policy. Our trial indicates that the intervention can lead to benefits in perceived psychological wellbeing, anxiety, and self efficacy for participants with osteoarthritis; however, although these psychological benefits were statistically significant, the effects were small and their clinical relevance for the population tested as a whole is unclear. Further work is needed to establish any predictive factors that might indicate participants who are most likely to benefit. Larger effects may be more likely in volunteers with high levels of motivation and morbidity.

Importantly, despite the enthusiasm of nurses based in the practices, it was often difficult to recruit patients to a self management programme similar to that advocated in government initiatives to empower patients. This suggests that patients may not perceive a need for such programmes.

\section{Indications for further research}

The main impact of this complex intervention was on psychological outcomes, but the process by which this was achieved is unclear. Further research is needed to clarify the mechanism whereby the intervention can effect change. An 
understanding of how the various components of the intervention may impact on different outcomes could allow appropriate targeting of patients to the intervention. This is important given the heterogeneity of the patient population receiving a generic self management approach to chronic disease (www.expertpatients.nhs.uk/).

\section{Conclusions}

Our study adds greatly to the literature on self management programmes for arthritis by reporting findings from a large randomised controlled trial of patients with osteoarthritis recruited from primary care and by using established and well validated outcome measures. Little doubt exists that such techniques provide some benefit for those with chronic conditions, but the best way to provide the intervention is unclear, and insufficient evidence exists to justify a policy of active recruitment of patients from primary care settings.

Thanks to Jennifer Beecham, Angela Coulter, and Melissa Pickering for help in conducting this trial; Peter Croft, Paul Dieppe, and Andrew Nunn for comments on an earlier draft; and Mike Kenward for statistical advice. Many thanks also to the MRC general practice framework nurses and general practices involved and all the patients who took part.

Contributors: MB, GR, MG, AP, JB, and AH helped conceive, design, and run the trial. IN and AA helped with the ongoing management of the trial and AA supervised data collection. MG had full access to all data and takes responsibility for the integrity of the data and the accuracy of the data analysis. All authors helped draft the paper and revise it critically and approved the final version. $\mathrm{MB}$ is guarantor.

Funding: Medical Research Council (reference G9900306).

Conflict of interest: None declared.

Ethical approval: Multicentre research ethics committee approval from the London office (reference MREC/99/2/87). Local research ethics committee approval was obtained in all areas where patients were recruited to the trial.

\section{What is already known on this topic}

Self management programmes for arthritis tested on US and UK volunteers indicate benefits to pain, depression, exercise taken, communication with doctors, and participants' perception of their ability to manage the disease

The government is promoting the widespread provision of a generic self management programme for chronic disease through primary care

\section{What this study adds}

Participants recruited from UK primary care to a randomised controlled trial of self management for arthritis reported reduced symptoms of anxiety and improved self efficacy in managing symptoms

The intervention had no significant effect on pain, physical functioning, or primary care contacts at 12 months' follow-up

These modest benefits do not justify a policy of active recruitment in primary care
1 Khaltaev N, Pfleger B, Woolf AD, Mathers C, Akesson K, Hazes JM, et al. Assessing the burden of musculoskeletal conditions: a joint World Health Organization-bone and joint decade project. Arthritis Res Ther 2003;5(suppl 3):174.

2 ARC. Factfile. Arthritis at a glance. www.arc.org.uk/about_arth/astats.htm http:// www.arc.org.uk/about_arth/astats.htm(last accessed 27 Jun 2005).

3 Lorig KR, Mazonson PD, Holman HR. Evidence suggesting health education for selfmanagement in patients with chronic arthritis has sustained health benefits while reducing health care costs. Arthritis Rheum 1993;36:439-46.

4 Barlow JH, Williams B. Improving arthritis self-management among older adults: "Just what the doctor didn't order." Br J Health Psychol 1997;2:175-85.

5 Barlow J, Wright C, Sheasby J, Turner A, Hainsworth J. Self-management approaches Barlow J, Wright C, Sheasby J, Turner A, Hainsworth J. Self-management approa
for people with chronic conditions: a review. Patient Educ Counsel 2002;48:177-87.

6 Solomon D, Warsi A, Brown-Stevenson T, Farrell M, Gauthier S, Mikels D, et al. Does self-management education benefit all populations with arthritis? A randomised controlled trial in a primary care physician network.J Rheumatol 2000;29:2:362-8.

7 Lorig K, Fries JF. The arthritis helpbook: a tested self-management program for coping with arthritis and fibromyalgia. 5th ed. Cambridge, Massachusetts: Perseus Books, 2000.

8 Barlow JH, Pennington DC, Bishop PE. Patient education leaflets for people with rheumatoid arthritis: a controlled study. Psychol Health Med 1997;2:221-35.

9 Jenkinson C, Wright L, Coulter A. Criterion validity and reliability of the SF 36 in a population sample. Qual Life Res 1994;3:7-12.

10 Sun Y, Sturmer T, Gunther KP, Brenner H. Reliability and validity of clinical outcome measurements of osteoarthritis of the hip and knee-a review of the literature. Clin Rheumatol 1997;16:185-98

11 Lorig K, Chastain R, Ung E, Shoor S, Holman HR. Development and evaluation of a scale to measure perceived self-efficacy in people with arthritis. Arthritis Rheum 1989;32:37-44.

12 Zigmond A, Snaith R. Hospital anxiety and depression scale. Acta Psychiatr Scand 1983;67:361-70.

13 Jenkinson C, Coulter A, Wright L. Short form 36 (SF36) health survey questionnaire: normative data for adults of working age. BMJ 1993;306:1437-40.

14 Barlow JH, Turner AP, Wright CC. Long-term outcomes of an arthritis self-management programme. Br J Rheumatol 1998;37:1315-9.

15 Vickers AJ, Altman DG. Analysing controlled trials with baseline and follow-up measurements. BMJ 2001;323:1123-4.

16 Little RJA, Rubin DB. Statistical analysis with missing data. 2nd ed. New York:John Wiley and Sons, 2002.

17 Schafer JL. Multiple imputation: a primer. Stat Methods Med Res 1999;8:3-15.

18 Lorig K, Holman H. Arthritis self-management studies: a twelve year review. Health Educ Quart 1993;20:17-28.

19 Bodenheimer T, Lorig K, Holman H, Grumbach K. Patient self-management of chronic disease in primary care. JAMA 2001;288:2469-75.

20 Warsi A, Wang P, LaValley M, Avorn J, Solomon DH. Self-management education programs in chronic disease: a systematic review and methodological critique of the literature. Arch Intern Med 2004;1641-9.

21 Lin EH, Katon W, Von Korff M, Tang L, Williams JW Jr, Kroenke K, et al. Effect of improving depression care on pain and functional outcomes among older adults with arthritis: a randomized controlled trial. JAMA 2003;290:2428-9.

22 Census 2001. www.statistics.gov.uk/cci/nugget.asp?id $=874$ and www.statistics.gov.uk/ $\mathrm{cci} /$ nugget.asp?id $=877$ (last accessed 27 Jun 2005)

23 Letter from the chief medical officer Sir Liam Donaldson to all primary care trust chief executives, $16 \mathrm{Feb} 2005$. www.dh.gov.uk/assetRoot/04/10/71/00/04107100.pdf (last accessed 27 Sep 2006).

(Accepted 24 August 2006)

doi $10.1136 /$ bmj.38965.375718.80

Department of Primary Care and Population Sciences, Royal Free and University College Medical School, London N19 5LW

Marta Buszewicz senior lecturer

Mark Griffin Statistician

Department of Primary Care and Population Sciences, Royal Free and University College Medical School, London NW3 2NF

Greta Rait senior lecturer

Irwin Nazareth professor

Centre for the Economics of Mental Health, Institute of Psychiatry, King's College London, London SE5 8AT

Anita Patel health economist

Clinical Research Facility, Royal Victoria Infirmary, Newcastle upon Tyne NE1 4LP Angela Atkinson project manager

Interdisciplinary Research Centre in Health, Coventry University, Coventry CV1 $5 \mathrm{FB}$

Julie Barlow professor of health psychology

London School of Hygiene and Tropical Medicine, London WC1E 7HT

Andy Haines dean

Correspondence to: M Buszewicz m.buszewicz@pcps.ucl.ac.uk 